\title{
Hyperinsulinaemic Hypoglycaemia and Polycystic Kidney Disease - A Rare Case Concerning PMM2 Gene Pleiotropy
}

\author{
Ana Rita Soares, ${ }^{1}$ Catarina Matos Figueiredo, ${ }^{2}$ Dulce Quelhas, ${ }^{3,4}$ Ermelinda Santos Silva, ${ }^{5}$ Joana Freitas, ${ }^{2}$ \\ Maria João Oliveira, ${ }^{2}$ Sameiro Faria, ${ }^{6}$ Ana Maria Fortuna ${ }^{1,4}$ and Teresa Borges ${ }^{2}$ \\ 1. Medical Genetics Department, Centre of Medical Genetics Doutor Jacinto Magalhães, Porto University Hospital Centre, Porto, Portugal; \\ 2. Paediatric Endocrinology Unit, Northern Maternal and Child Centre, Porto University Hospital Centre, Porto, Portugal; 3. Biochemical Genetics \\ Unit, Centre of Medical Genetics Doutor Jacinto Magalhães, Porto University Hospital Centre, Porto, Portugal; 4. Unit for Multidisciplinary \\ Research in Biomedicine, Abel Salazar Biomedical Sciences Institute, Porto University, Porto, Portugal; 5. Paediatric Gastroenterology Unit, \\ Northern Maternal and Child Centre, Porto University Hospital Centre, Porto, Portugal; 6. Paediatric Nephrology Unit, Northern Maternal and \\ Child Centre, Porto University Hospital Centre, Porto, Portugal
}

DOI: https://doi.org/10.17925/EE.2020.16.1.66

\begin{abstract}
$\mathrm{C}$ o-occurrence of hyperinsulinaemic hypoglycaemia and polycystic kidney disease (HIPKD) has been recently described. It is caused by a non-coding variant in the promoter region for phosphomannomutase 2 (PMM2), C.-167G>T, both in homozygous or compound heterozygous variants with deleterious coding. Although PMM2 has been associated with congenital disorder of glycosylation, patients do not present with this phenotype and have normal carbohydrate-deficient transferring testing. The authors present a rare case where specific PMM2 study was performed as a result of clinical suspicions. The patient was a 6-year-old female followed at our clinic due to congenital hyperinsulinism since she was 1 month old. She also presented with bilateral polycystic kidneys, detected in prenatal set, and simple hepatic cysts, for which she was treated with diazoxide and captopril. Initial metabolic and genetic studies were normal. PMM2 gene sequence study revealed the promotor variant c.-167G>T in compound heterozygosity with the previously described pathogenic variant c.422G >A (p.Arg141His), confirming the diagnosis of HIPKD. This is a notable case as it highlights the importance of keeping this diagnostic hypothesis in mind and serves as a reminder to perform proper clinical and genetic investigation. A correct, and early, diagnosis will avoid unnecessary additional investigations and will allow appropriate genetic counselling for this autosomal recessive disorder.
\end{abstract}

\section{Keywords}

Hyperinsulinemic hypoglycemia, polycystic kidney disease, PMM2 gene

Disclosures: Ana Rita Soares, Catarina Matos Figueiredo, Dulce Quelhas, Ermelinda Santos Silva, Joana Freitas, Maria João Oliveira, Sameiro Faria, Ana Maria Fortuna and Teresa Borges have no financial or non-financial relationships or activities to declare in relation to this article.

Review Process: Double-blind peer review.

Compliance with Ethics: Informed consent was received from the parents of the patient involved in this case study. No identifying information or images have been included.

Authorship: All named authors meet the criteria of the International Committee of Medical Journal Editors for authorship for this manuscript, take responsibility for the integrity of the work as a whole and have given final approval for the version to be published. Access: This article is freely accessible at touchENDOCRINOLOGY.com @ C Touch Medical Media 2020. Received: 26 August 2019

Accepted: 8 November 2019

Published Online: 4 February 2020

Citation: European Endocrinology. 2020;16(1):66-8

Corresponding Author: Ana Rita Soares, Medical Genetics Department, Centre of Medical Genetics Doutor Jacinto Magalhães, Porto University Hospital Centre Address: Largo da Maternidade Júlio Dinis, Serviço de Genética Médica, Piso 1, 4050-371, Porto,

Portugal. E: anaritamsoares@gmail.com

Support: No funding was received in the publication of this article.
Hyperinsulinaemic hypoglycaemia $(\mathrm{HH})$ is a very rare disease with an estimated incidence of $1 / 50,000,{ }^{1}$ and includes a clinical, histological and genetic heterogeneous group of disorders characterised by hypoglycaemia, hyperinsulinism or, more specifically, increased C-peptide level and inappropriate low blood levels of ketone bodies during a hypoglycaemic event. $\mathrm{HH}$ is a variable condition: it can be transient or persistent; patients can present with mild symptoms or with very symptomatic hypoglycaemia with adrenergic symptoms or life-threatening neurologic symptoms; it can be congenital or secondary, mainly to overgrowth syndromes/metabolic disorders. Pancreatic lesions that can cause $\mathrm{HH}$ may be diffuse or focal. Both 18F-fluoro-dihydroxyphenylalanine (18F-DOPA) positron emission tomography (PET)/computed tomography (CT) and genetic study are essential diagnostic tools to differentiate these types, with important management implications. Until 2017, 12 genes were described in the literature associated with $\mathrm{HH}$, but still $50 \%$ of cases remain without genetic confirmation. ${ }^{2}$ Management depends on both phenotype and genotype, but all require intravenous glucose at very high infusion rate ( $>8 \mathrm{mg} / \mathrm{kg} / \mathrm{min}$ ) at presentation. There are different approaches for management in the non-acute phase, such as increased glucose intake (oral or intravenous), dietary modifications, exercise eviction, pharmacological treatment (such as diazoxide, octreotide or lanreotide), hormone administration (such glucagon) or surgical treatment (excision of focal lesion or almost total pancreatectomy for diffuse lesion).

Polycystic kidney disease (PKD), mainly the autosomal recessive type, is a hepatorenal fibrocystic disease, ${ }^{3}$ characterised by bilaterally enlarged, diffusely echogenic kidneys that can be found on prenatal ultrasound or after birth, and biliary ductal ectasia or signs of congenital hepatic fibrosis. It is usually more severe than the autosomal dominant type and presents earlier in life. Biallelic pathogenic variants in the PKHD1 gene are found in about $42-87 \%$ of patients. ${ }^{3}$ About $30 \%$ of these children die in the neonatal period or within the first year of life due to respiratory insufficiency consequent to oligohydramnios. ${ }^{4}$ The co-occurrence of both diseases (HIPKD) has been recently described by Cabezas et al., in a group of 17 patients from 11 families. ${ }^{5}$ It is caused by non-coding variant in the promoter region for phosphomannomutase 2 (PMM2), C.-167G>T, both in homozygous or compound heterozygous variants with deleterious coding. 
Here we present a new case of this rare entity recently diagnosed in a central hospital in Portugal.

\section{Case presentation}

The patient was a 6-year-old female, the second child of a healthy non-consanguineous couple, followed at our paediatric endocrinology outpatient clinic due to congenital hyperinsulinism since she was 1 month old. She was born after a well-monitored and uneventful pregnancy without gestational diabetes, but with a prenatal diagnosis of bilateral renal hypertrophy. She was born by vaginal delivery at 36 weeks of gestation, with weight 3,860 g (Z-score 2.55 P99), length $47 \mathrm{~cm}$ (Z-score 0.21 P58) and head circumference $35 \mathrm{~cm}$ (Z-score 1.88 P97). She presented with palpable flank masses of hard consistency, without other manifestations on perinatal observation. At 20 hours of life, she presented with hypotonia and hypoglycaemia and was admitted to the neonatal intensive care unit (NICU) with severe hypoglycaemia (4 mg/dL), high insulin levels $(19.2 \mu \mathrm{U} / \mathrm{mL})$ and increased serum lactate $(3.01 \mathrm{mmol} / \mathrm{L})$. Lactate values were posteriorly normal. The last one was normal on the following measurements. At NICU, she developed hypertension at 7 days old and had persistent tendency to hypoglycaemia despite progressive increase in glucose rate (maximum of $17.6 \mathrm{mg} / \mathrm{kg} / \mathrm{min}$ IV and milk plus maltodextrin). She had feeding problems requiring a nasogastric tube feeding to complement breastfeeding. Initially she needed to be fed every 2 hours to reach a good metabolic control (blood glucose $\geq 60 \mathrm{mg} / \mathrm{dL}$ ).

An ultrasound revealed patent foramen ovale, slight hypertrophy of the left ventricle and minimal aortic insufficiency. Kidney and abdominal echography revealed very globose kidneys, with increased bipolar diameter (right kidney with $63 \mathrm{~mm}$ and left kidney with $67 \mathrm{~mm}$, both >P95 for age), attenuation of parenchymal differentiation and presence of multiple pericentimetric cysts, as well as some simple hepatic cysts with infra- and pericentimetric dimensions. Extensive metabolic study, including urinary organic acids, 17-dihydrocholesterol in dried blood spot, plasma very long chain fatty acids, lactate, ammonia, acylcarnitine profile and carbohydrate-deficient transferring, turned out to be normal. On day 23 , she was started on diazoxide with consequent glycaemia stabilisation and subsequent progressive reduction of glucose load. At day 37, she was discharged home with diazoxide ( 9 mg/kg/day) and captopril (0.2 mg/day).

Subsequently, during follow-up in the endocrinology clinic, several genetic studies were performed, including karyotype analysis, array-based comparative genomic hybridisation, methylationspecific multiplex ligation-dependent probe amplification for Beckwith-Wiedemann syndrome, ABCC8 and KCNJ11 genes for congenital hyperinsulinism and PKHD1 for autosomal recessive PKD, all without relevant alterations.

The child grew at normal rates for both weight and height and had neither dysmorphic nor neurologic features. Cognitive evaluation was performed at 55 months and revealed a normal developmental quotient (113 for Griffith Mental Development Scale). ${ }^{6}$

At the time of this case report, the child was 6 years and 5 months old, and is still on diazoxide treatment with normoglycaemia. Her blood sugars are well-controlled on diazoxide, as measured by continuous glucose monitoring. She has normal blood pressure on captopril treatment, with her last echocardiogram evaluation showing no structural cardiopathy and no left ventricular hypertrophy with a good ventricular function. Her last glomerular filtration rate was
$70 \mathrm{~mL} / \mathrm{min} / 1.73 \mathrm{~m}^{2}$ and liver transaminases levels were normal (aspartate aminotransferase [AST] $31 \mathrm{UI} / \mathrm{L}$ and alanine aminotransferase [ALT] $18 \mathrm{UI} / \mathrm{L})$. Her last abdominal and renal ultrasound still revealed enlarged kidneys (right kidney $111 \mathrm{~mm}$ and left kidney $117 \mathrm{~mm}$, both >95 centile for age) with multiples small cysts and multiple hepatic cysts without liver enlargement.

Looking for the possible underlying cause of this phenotype, study of the PMM2 gene sequence was performed, specially searching for promotor variant C. $-167 \mathrm{G}>\mathrm{T}$. This study revealed that our patient had both this pathogenic variant and the pathogenic variant c.422G $>$ A (p.Arg141His) in compound heterozygosity. R141H (NM_000303.2: c.422G>A; NP_000294.1: p.Arg141His) is the most frequent mutation and was detected in 8 out of 1,000 in a default global population (1,000 genome phase 1 genotype data from 1,094 worldwide individuals). ${ }^{7} \mathrm{R} 141$ is located in the active site of the enzyme where it is predicted to bind the phosphate group of the substrate, mannose-6-phosphate.? Its mutation into histidine, which completely inactivates the enzyme, has never been observed in homozygosity, possibly because the total absence of PMM2 is not compatible with life. ${ }^{8}$ This result confirmed the diagnosis of HIPKD.

In genetic consultation, parental carrier status was confirmed and appropriate genetic counselling was given to the family.

\section{Discussion}

HIPKD is a rare disease and, as far as the authors know, there is only one original paper describing a group of patients that presented with this phenotype and in whom the causative variant was found through Whole Exome sequencing (WES) in the research set. ${ }^{5}$ HIPKD is caused by a non-coding variant in the promoter region for PMM2, C.-167G>T, both in homozygous or compound heterozygous variants with previously described deleterious coding.

PMM2 is a key enzyme in N-glycosylation, and recessive coding pathogenic variants in PMM2 cause a congenital disorder of glycosylation PMM2-CDG, formerly known as CDG type 1a. This is a multisystemic and variable disorder characterised mainly by cerebellar dysfunction, developmental delay, abnormal subcutaneous fat distribution and inverted nipples, strabismus and hypotonia, and other clinical manifestations. Transferrin isoelectric focusing study reveals an abnormal pattern type 1 and the diagnosis is confirmed by Sanger sequencing of PMM2 gene. For patients to present with PMM2-CDG type, two pathogenic coding variants should be found. However, patients described with HIPKD have this specific promotor variant in PMM2 and do not present multisystemic involvement of the disease, neither have abnormal transferrin isoelectric focusing. ${ }^{5}$

This is the first case where the recent knowledge from Cabezas et al. ${ }^{5}$ was applied in the diagnostic work-up. Comparing with cases described by Cabezas et al., persistent hyperinsulinism needing diazoxide treatment was described in 9/17 (53\%) patients; kidney anomalies were found previously in 17/17 (100\%) and hepatic cystic disease was described in $6 / 17$ (35\%) patients. The molecular study in this case report confirmed the previously described variant associated with this phenotype. ${ }^{5}$ It is an autosomal recessive disorder, with $25 \%$ risk of recurrence for this child's parents, and appropriate genetic counselling was performed. ${ }^{5}$

The authors wish to emphasise that it is essential to keep this diagnostic hypothesis in mind. If prenatal renal cysts are detected, it is important to measure blood glucose and insulin after birth; if a 


\section{Case Report Diabetes}

patient presents with persistent $\mathrm{HH}$, performing a renal/abdominal ultrasound should be considered. If this clinical diagnosis is suspected, specific sequencing of PMM2 gene should be performed, with special attention to the promoter region. A correct and early diagnosis will avoid unnecessary additional investigations and will allow appropriate genetic counselling for this autosomal recessive disorder. $\square$
1. Mohmed Z, Arya VB, Hussain K. Hyperinsulinaemic hypoglycaemia: genetic mechanisms, diagnosis and management. J Clin Res Pediatr Endocrinol. 2012;4:169-81.

2. Demirbilek $\mathrm{H}$, Hussain $\mathrm{K}$. Congenital hyperinsulinism: diagnosis and treatment update. I Clin Res Pediat Endocrinol. 2017;9(Suppl. 2):69-87.

3. Gunay-Aygun M, Tuchman M, Font-Montgomery E, et al. PKHD1 sequence variations in 78 children and adults with autosomal recessive polycystic disease and congenital hepatic fibrosis. Mol Genet Metab. 2010:99:160-73. Sweeney WE, Avner ED. Polycystic Kidney Disease, Autosomal Recessive. 2001 July 19 [Updated 2019 Feb 14]. In: Adam MP. Ardinger $\mathrm{HH}$, Pagon RA, et al., editors. GeneReviews ${ }^{\circledast}[$ Internet]. Seattle (WA): University of Washington, Seattle.

5. Cabezas OR, Flanagan SE, Stanescu H, et al. Polycystic kidney disease with hyperinsulinemic hypoglycemia caused by promoter mutation in phosphomannomutase 2. J Am SOC Nephrol. 2017;28:2529-39.
6. Green E, Stroud L, Bloomfield, et al. Griffiths Scales of Child Development, Third Edition. Oxford, UK: Hogrefe, 2015.

7. Andreotti G, Cabeza de Vaca I, Poziello A, et al. Conformational Andreoti G, Cabeza de vacal, Pozillo A, et al. Conformational response to ligand binding in phosphomannomutase2: Insights
into inborn glycosylation disorder. J Biol Chem. 2014;289:34900-10

8. Matthijs G, Schollen E, Van Schaftingen $E$, et al. Lack of homozygotes for the most frequent disease allele in carbohydrate-deficient glycoprotein syndrome type $1 \mathrm{~A}$ Am J Hum Genet. 1998;62:542-50. 